\title{
Adiciones a la flora briofítica del Espacio Natural Sierra de las Nieves (Málaga, España)
}

\author{
Juan Guerra', Federico Casimiro-Soriguer Solanas ${ }^{2}$ \& Baltasar Cabezudo² \\ ${ }^{1}$ Área de Botánica (Dpto. de Biología Vegetal), Facultad de Biología, Universidad de Murcia, Campus de Espinardo, Murcia \\ 2 Área de Botánica (Dpto. de Botánica y Fisiología Vegetal), Facultad de Ciencias, Universidad de Málaga, Campus de \\ Teatinos, Málaga
}

\section{Correspondencia}

\section{J. Guerra}

email: jguerra@um.es

Recibido: 19 octubre 2020

Aceptado: 26 octubre 2020

Publicado on-line: 3 noviembre 2020

Editado por: Marta Recio Criado

\begin{abstract}
Resumen
Se citan 14 especies de briofitos nuevas para el Espacio Natural Sierra de las Nieves, elevándose a 257 el total de especies localizadas en el territorio. Destacamos la presencia de dos especies de Schistidium recientemente descritas y de Neckera cephalonica Jur. \& Unger y Eurhynchiastrum pulchellum (Hedw.) Ignatov \& Huttunen, taxones muy raros en el sur de la Península Ibérica.
\end{abstract}

Palabras clave: Flora briofítica, Andalucía, Espacios protegidos, Parque Nacional.

\section{Abstract}

Additions to the bryophyte flora of the Sierra de las Nieves Natural Area (Málaga, Spain).

Fourteen new species of bryophytes are recorded for the Sierra de las Nieves Natural Area, raising the total number of species located in the territory to 257 . We highlight the presence of two recently described Schistidium species, and Neckera cephalonica Jur. \& Unger and Eurhynchiastrum pulchellum (Hedw.) Ignatov \& Huttunen, very rare taxa in southest Iberian Peninsula.

Key words: Bryophyte flora, Andalusia, Protected Natural Area, National Park.
En el marco de un proyecto sobre la flora del Espacio Natural Sierra de las Nieves (Málaga, España), se publicaron los resultados de 2 años de prospecciones (2017-2018) sobre la brioflora del territorio (Guerra et al. 2018). En este trabajo se citaron un total de 243 especies de briófitos (208 musgos, 32 hepaticas y 3 antocerotas), lo que puede considerarse una riqueza en especies de briófitos excepcional en el sur de la Península Ibérica. Estos datos ya podrían justificar una figura de protección para la zona bajo la figura de Parque Nacional, pues en Andalucía sólo el Parque Nacional de Sierra Nevada (Granada) o el Parque Natural de Cazorla, Segura y las Villas presentan un grado de biodiversidad briofítica comparable.

Como era de esperar, producto de nuevos trabajos de campo durante los años 2019-2020, se aportan nuevos hallazgos que se recopilan en el presente artículo. Algunos de estos tienen cierta relevancia, a destacar la presencia de dos nuevas especies de Schistidium recientemente descritas (Guerra et al. 2019, 2020) o Neckera cephalonica Jur. \& Unger y Eurhynchiastrum pulchellum (Hedw.) Ignatov \& Huttunen, taxones muy raros en el sur de la Península. Por el momento, el número de especies de briófitos conocidas en el Espacio Natural Sierra de las Nieves, del que forma parte el futuro Parque Nacional Sierra de las Nieves, se eleva a 257. Se han estudiado un total de 2579 muestras recolectadas durante los años 2017 a 2020, que se encuentran depositadas en los herbarios de la Universidad de Málaga (MGC) y Universidad de Murcia (MUB).

\section{Resultados}

\section{Bryopsida (Musgos)}

\section{POLYTRICHACEAE}

Pogonatum nanum (Hedw.) P. Beauv.

ESPAÑA. Málaga, Parauta, Sierra de las Nieves, alrededores del Peñón del Robledal, 30SUF1656, 1200 m, 7-III-2019, Cabezudo \& Soriguer (MUB 58322, MGC 39569).

Este taxon es recogido por Cano \& Guerra (2020) como una novedad para la flora de la provincia de Málaga. Ha sido encontrado en suelos de naturaleza silícea. 


\section{FISSIDENTACEAE}

Fissidens pusillus (Wilson) Milde

ESPAÑA. Málaga, Yunquera, Sierra del Pinar, cuevas de los Tajos de la Alberca, 30SUF2349066123, 1350 m, 11-VI-2020, Cabezudo \& Soriguer (MGC 4793).

Se encuentra frecuentemente en lugares salpicados por el agua o muy húmedos, en la entrada de cuevas, a veces en tobas calizas, tal y como ha aparecido en la zona. Guerra \& Ederra (2015) recogen que ha sido citada en la provincia de Málaga, pero sin material de referencia que se hubiese podido estudiar. Se confirma, por tanto, la presencia en esta provincia.

\section{GRIMMIACEAE}

Schistidium convergens J. Guerra \& M.J. Cano

ESPAÑA. Málaga, Istán, Cerro del Duque, pista desde el puerto de la Refriega al puerto del Algarrobo, 30SUF19377/55950, 900-1500 m, 29-VI-2017, Cabezudo \& Soriguer (MUB 56094).

Especie descrita recientemente del norte de Africa y Sierra Nevada (Guerra et al. 2019). Se trata de una especie acidófila, relativamente rara incluso en las zonas de donde se ha descrito. En la Sierra de las Nieves se encuentra sobre peridotitas.

\section{Schistidium memnonium J. Guerra}

ESPAÑA. Málaga, Ronda, Sierra de las Nieves, entre el Cortijo de Quejigales y el cerro de la Yedra, 30SUF1762, 1300 m, 29-V-2018, Cabezudo \& Soriguer (MUB 56953).

Taxon circunmediterráneo que llega a zonas cálidas del centro de Europa (cf. Guerra et al. 2020). Es una especie que vive a cierta altitud en rocas calizas o dolomíticas expuestas, tal y como se ha encontrado en la zona.

\section{POTTIACEAE}

\section{Syntrichia calcicola J.J. Amann}

ESPAÑA. Málaga, Parauta, cerro Abanto, vereda desde el puerto del Robledal a puerto del Capuchín, 30SUF1675, 1280 m, 7-III-2019, Cabezudo \& Soriguer (MGC 3970).

Especie relativamente rara en el territorio estudiado, que no había sido citada con anterioridad en la provincia de Málaga (cf. Gallego 2006). Ha aparecido colonizando suelos sobre rocas peridotíticas.

Tortula acaulon var. pilifera (Schreb. ex Hedw.) R.H. Zander

ESPAÑA. Málaga, Parauta, Sierra de las Nieves, alrededores del Peñón del Robledal, 30SUF1656, 1200 m, 7-III-2019, Cabezudo \& Soriguer (MUB 56729, MGC 3966).

Citada como novedad para la flora de Málaga (Cano \& Guerra 2020), se trata de un taxón relativamente raro en la Península Ibérica y muy raro en el territorio del futuro Parque Nacional.

\section{NECKERACEAE}

Alleniella besseri (Lobarz.) S. Olsson, Enroth \& D. Quandt

(= Neckera besseri (Lobarz.) Jur.)

ESPAÑA. Málaga, Tolox, Sierra de las Nieves, quejigal de Tolox, vereda al Peñón de los Enamorados, 30SUF2173, 1650 m, 5-VI-2019, Cabezudo \& Soriguer (MUB 57886, MGC 4354).

Cano \& Guerra (2020) la citan como novedad para la provincia de Málaga. En el territorio estudiado aparece en lugares sombríos, en el fondo de torcas.

\section{Neckera cephalonica Jur. \& Unger}

ESPAÑA. Málaga, Parauta, Sierra de las Nieves, Peñón del Robledal, 30SUF1656, 1350 m, 7-V-2019, Cabezudo \& Soriguer (MUB 58788, MGC 4677).

Especie infrecuente en áreas mediterráneas, salvo en ombroclimas húmedos. Es frecuente, sin embargo, en territorios cercanos del Parque de los Alcornocales (Tarifa, Algeciras). La localidad que se cita es la única que conocemos en el territorio estudiado. En la zona estudiada se ha localizado sobre tronco de Quercus pyrenaica Willd.

\section{BRACHYTHECIACEAE}

\section{Brachythecium rivulare Schimp.}

ESPAÑA. Málaga, Igualeja, Sierra de las Nieves, vereda de la Fuenfría, Peñón del Robledal, arroyo del Realejo, 30SUF1656, 1200-1300 m, 9-VI-2017, Cabezudo \& Soriguer (MGC 2964).

Taxón muy frecuente en toda la Península Ibérica, coloniza rocas sumergidas o salpicadas en ríos, arroyos, manantíos, etc. Sin embargo, no es frecuente en el territorio que aquí se estudia. En la zona ha aparecido sobre gneises en las rocas de un arroyo.

\section{Eurhynchiastrum pulchellum (Hedw.) Ignatov \& Huttunen}

ESPAÑA. Málaga, Parauta, río Grande, Los Manaderos, 30SUF1262, 915 m, 7-III-2019, Cabezudo \& Soriguer (MGC 3943). Málaga, El Burgo, Convento de las Nieves, nacimiento de Huerta Alta, 30SUF2469, 700 m, 21-III-2019, Cabezudo \& Soriguer (MGC4028). Málaga, Tolox, Sierra de las Nieves, cerro Pilones, 30SUF19296/62152, 1745 m, fondo de simas (-15 m), 24-X-2019, Cabezudo \& Soriguer (MGC4720).

Especie relativamente rara en el sur de la Península lbérica, se suele encontrar a cierta altitud en hendiduras de rocas calizas con suelo, generalmente en taludes húmedos y sombríos. En la zona se ha encontrado ocasionalmente en el fondo de simas En la provincia de Málaga se conoce también de la Sierra de la Almijara (Guerra 2018).

Microeurhynchium pumilum (Wilson) Ignatov \& Vanderp.

ESPAÑA. Málaga, Tolox, Sierra de las Nieves, quejigal de Tolox, sima del Nevero, $(-10 \mathrm{~m})$, 30SUF21861/63900, 1650 m, 22-V-2019, Cabezudo \& Soriguer (MGC 4633, MGC 4641). 
Extendida por toda la Península lbérica. Se ha encontrado en la zona colonizando paredes y suelos de simas.

Plasteurhynchium striatulum (Spruce) M. Fleisch.

ESPAÑA. Málaga, Tolox, Sierra de las Nieves, quejigal de Tolox, sima del Nevero, $(-10 \mathrm{~m})$, 30SUF21861/63900, 1650 m, 22-V-2019, Cabezudo \& Soriguer (MGC 4638, MUB 59379).

Extendida por la Península lbérica. Hacia el sur es relativamente frecuente en lugares de alta montaña caliza, en situaciones muy sombrías. En la zona se ha encontrado en las rocas verticales del fondo de una sima.

\section{SEMATOPHYLLACEAE}

\section{Sematophyllum substrumulosum (Hampe) E. Britton}

ESPAÑA. Málaga, Istán, Sierra de las Apretaderas, pista del Meliche al río Hoyo del Bote, vado del río, 30SUF22302/50376, 340 m, 7-V-2019, Cabezudo \& Soriguer (MUB 57821).

Se recoge en el trabajo de Cano \& Guerra (2020) como una novedad para la provincia de Málaga, constituyendo el límite oriental de la especie en el territorio peninsular. En la localidad conocida en la zona aparece colonizando troncos de lentisco (Pistacia lentiscus L.)

\section{HYPNACEAE}

\section{Hypnum uncinulatum Jur.}

ESPAÑA. Málaga. Benahavís, Sierra Palmitera, cuenca del río Guadaiza, Tajos del Mirador, 30SUF18618/49500, 655 m, Cabezudo \& Soriguer, 15-III-2019 (MGC 3995).

Aunque es relativamente frecuente en la mitad occidental de la Península Ibérica, bajo clima mediterráneo-atlántico (Ríos et al. 2018), es rara en la zona, donde ha aparecido colonizando rocas ácidas (gneises) en taludes. Esta es la primera localidad conocida en la provincia de Málaga.

\section{Agradecimientos}

Este trabajo se enmarca en el convenio Junta de
Andalucía-Grupo de Investigación RNM-115 de la Universidad de Málaga para el "Estudio de la Flora y Vegetación del futuro Parque Nacional Sierra de las Nieves". Agradecemos a María J. Cano y M. Teresa Gallego (Universidad de Murcia) su ayuda en la revisión de algunas muestras conflictivas. Agradecemos a Rogelio Ferrer (Federación Andaluza de Espeleología) la localización de alguna de las simas estudiadas en este trabajo.

\section{Bibliografía}

Cano, M.J. \& Guerra, J. (2020). Novedades corológicas para la flora briofítica ibérica. IX. Anales de Biología 42, 1-7.

Gallego, M.T. (2006). Syntrichia Brid. In Guerra, J., Cano, M.J. \& Ros, R.M. (eds.). Flora Briofítica Ibérica, vol. 3: 120-143. Universidad de Murcia /SEB, Murcia.

Guerra, J. (2018). Eurhynchiastrum Ignatov \& Huttunen. In Guerra, J., Cano, M.J. \& Brugués, M. (eds.). Flora Briofítica Ibérica, vol. VI: 186-190. Universidad de Murcia/SEB, Murcia.

Guerra, J. \& Ederra, A. (2015). Fissidentaceae Lindb. In Guerra, J. \& Brugués, M. (eds.). Flora Briofítica Ibérica, vol. II: 153-187. Universidad de Murcia /SEB, Murcia.

Guerra, J., Gallego, M.T., Jiménez, J.A., Cano, M.J., Casimiro-Soriguer Solanas, F., Pérez Latorre, A.V. \& Cabezudo, B. (2018). Flora briofítica del espacio natural Sierra de las Nieves y su entorno (Málaga, España). Universidad de Murcia, Murcia.

Guerra, J., Jiménez-Martínez, J.F., Cano, M.J., Alonso, M., Gallego, M.T. (2019). Schistidium convergens (Grimmiaceae, Bryophyta), a new species from southern Spain and Morocco. Nova Hedwigia, 109: 65-80.

Guerra, J., Martínez, M., Jiménez, J.A., Cano, M.J. \& Gallego, M.T. (2020). A new species of moss emerged under DNA and morphological data: Schistidium memnonium sp. nov. (Grimmiaceae, Bryophyta). Plant Biosystems. doi.org/10.1080/11263504.2020.1762789.

Ríos, D., Gallego, M.T. \& Guerra, J. (2018). Hypnum Hedw. In Guerra, J., Cano, M.J. \& Brugués, M. (eds.). Flora Briofítica Ibérica, vol. VI: 336-367. Universidad de Murcia/SEB, Murcia. 